\title{
Oxidant and antioxidant parameters in people who fast during Ramadan, and those who do not
}

Hasan Karsen, Harran University Faculty of Medicine, Department of Infectious Diseases, Şanlıurfa, Turkey. drhasankarsen@gmail.com

Emine Ayca Güler, Harran University Faculty of Medicine, Department of Infectious Diseases, Şanlıurfa, Turkey.aycakdemir@yahoo.com

İrfan Binici, Harran University Faculty of Medicine, Department of Infectious Diseases, Şanlıurfa, Turkey. irfanbinici@gmail.com

Huseyin Taşkıran, Harran University Faculty of Medicine, Department of Internal Medicine, Şanlıurfa, Turkey. huseyintaskiran@hotmail.com

Suleyman Yıldırım, Halk Sağlığı Müdürlüğü, Gaziantep, Turkey. drsyildirim@gmail.com

İsmail Koyuncu, Harran University Faculty of Medicine, Department of Biochemistry, Şanlıurfa, Turkey.

ismailkoyuncu1@gmail.com

\section{Abstract \\ Background:}

Objectives: The main aim of this study was to compare, by a new automated colorimetric method, oxidant and anti-oxidant status of those who fast during Ramadan and those who do not. For this purpose, total oxidant status (TOS), total anti-oxidant capacity (TAC) levels and oxidative stress index (OSI) were analysed. By doing this, we hope to examine whether fasting has any beneficial effect on human health.

Materials and methods: Fifty seven people were included in this study. Of these, 30 people had been fasting during the month of Ramadan for at least ten years, but 27 had never fasted in their life. On the $15^{\text {th }}$ day of Ramadan, blood samples were obtained from both groups after 12 hours of fasting.

Results: The TAC levels of the fasting group $(1.29 \pm 0,19)$ was quite higher than the non-fasting group $(1,09 \pm 0,16)$ [EIC2] [CD3] [ICD4] $(\mathrm{p}<0.001)$. Similarly, in the fasting group TOS level and the OSI (respectively 12,77 $\pm 2,23$ and 1,01 $\pm 0,25)$ was quite lower [EIC5][ICD6][ICD7] than in the non-fasting group (respectively 14,15 $\pm 2,04$ and 1,33 $\pm 0,30),(\mathrm{p}<=0.019$ and $\mathrm{p}<0.001$, respectively).

These findings show that the TOS level of non-fasting group was high. This oxidative stress might cause various illnesses. Therefore, fasting could play a significant part in health-protection by increasing total anti-oxidant capacity.

Keywords: Fasting Ramadan, oxidative parameters.

DOI: https://dx.doi.org/10.4314/ahs.v19i3.46

Cite as: Karsen H, Güler EA, Binici I, Taşkıran H, Yuldrrm S, Koynncu I. Oxidant and antioxidant parameters in people who fast during Ramadan, and those who do not. Afri Health Sci. 2019;19(3): 2713-2717. https://dx.doi.org/10.4314/ahs.v19i3.46

\section{Introduction}

Allah orders in the Holy Book Quran, to people who believe, to fast during Ramadan ${ }^{1}$. Therefore, fasting during Ramadan is a necessity (fard) for all believers in Islam.

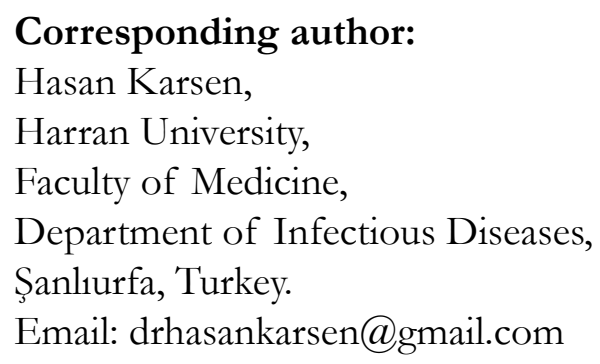

Similarly, the prophet of Islam, Mohammed (Peace Be Upon Him) recommends that "fast so that you find good bealth" ${ }^{2}$. In accordance with this recommendation, most of the Muslims fast 29 or 30 days consecutively during the month of Ramadan, which is the ninth month of Islamic lunar calendar, so its duration differs every year. In fasting days, believers do not eat anything from dawn to sunset ${ }^{3}$. There are many studies about the effect of Ramadan fasting on human health ${ }^{4,5}$. As far as we know, there is no study that compares the importance of oxidative stress index between fasting and non-fasting groups. In this study, we aimed at evaluating the oxidative status in people who fast during Ramadan and those who do

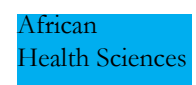

2713

(C) 2019 Karsen et al. Licensee African Health Sciences. This is an Open Access article distributed under the terms of the Creative commons Attribution License (https://creativecommons.org/licenses/BY/4.0), which permits unrestricted use, distribution, and reproduction in any medium, provided the original work is properly cited. 
not. Via a new automated colorimetric method, we have measured the total anti-oxidant capacity (TAC) and total oxidant status (TOS); and calculated the oxidative stress index. The measurement method developed by Erel, has many advantages when compared with other methods. It is simple, cheap, reliable, and precise. Moreover, it does not interact with commonly occurring serum components ${ }^{6,7}$.

\section{Materials and methods}

Fifty-seven people were included in this study. Of these, 30 people (13 female, 17 male) had been fasting during Ramadan for at least ten years, but 27 (16 female, 11 male) had never fasted in their life. On the $15^{\text {th }}$ day of the Ramadan, blood samples were obtained from both groups, on an empty stomach after 12 hours. Demographic features like age, gender, and Body Mass Index (BMI) of the both groups were matched. Total oxidant status (TOS) and total anti-oxidant capacity (TAC) levels were analysed via a new colorimetric method, developed by Erel. Oxidative stress index (OSI) was also calculated.

\section{Exclusion criteria}

Those who had the following health problems were not included in this study: smoking, chronic kidney disease, chronic obstructive pulmonary disease, hypertension, cardio-vascular diseases, malignancy, rheumatic diseases, chronic inflammatory diseases, infection, and degenerative diseases.

Measurement of the Total Antioxidant Capacity (TAC) TAC of serum was determined using a novel automated measurement method, developed by Erel $^{6}$. In this method, the hydroxyl radical, which is the most potent biological radical, is produced. In the assay, ferrous ion solution, which is present in Reagent 1 is mixed with hydrogen peroxide, which is present in the Reagent 2 . The sequential produced radicals such as brown coloured dianisidine radical cation, produced by the hydroxyl radical, are also potent radicals. Using this method, anti-oxidative effect of the sample is measured against the potent free radical reactions, which is initiated by the produced hydroxyl radical. The assay has got excellent precision values, which are lower than $3 \%$. The results are expressed as mmol Trolox Equiv./L.

\section{Measurement of Total Oxidant Status (TOS)}

TOS of serum was determined using a novel automated measurement method, developed by Erel ${ }^{7}$. Oxidants which are present in the sample oxidize the ferrous ion-o-dianisidine complex to ferric ion. The oxidation reaction is enhanced by glycerol molecules, which are abundant in the reaction medium. The ferric ion makes a coloured complex with xylenol orange in an acidic medium. The colour intensity, which can be measured spectrophotometrically, is related to the total amount of oxidant molecules present in the sample. The assay is calibrated with hydrogen peroxide and the results are expressed in terms of micro molar hydrogen peroxide equivalent per liter ( $\mu \mathrm{mol} \mathrm{H}_{2} \mathrm{O}_{2}$ Equiv./L).

\section{Oxidative Stress Index (OSI)}

The ratio of TOS to TAC yields the OSI, an indicator of the degree of oxidative stress ${ }^{6,7}$. For calculations, the resulting unit of TAC was changed to $\mathrm{mmol} / \mathrm{l}$, and the OSI value was calculated according to the following formula: OSI $($ arbitrary unit $)=$ TOS $\left(\mathrm{mmol} \mathrm{H}_{2} \mathrm{O}_{2}\right.$ equiv. $\left./ \mathrm{l}\right) /$ TAS (mmol Trolox equiv./l).

\section{Statistical analysis}

All of the analyses were conducted using the IBM SPSS 22.0 statistical program (Chicago, IL, USA). Data were expressed as mean \pm standard deviation. The normality of the distributions was evaluated using the Kolmogorov-Smirnov test. Comparisons of the parameters were performed using the Student's t-test. A $\chi^{2}$ test was used to compare gender distributions within groups. All of the statistical tests were two-sided; a $\mathrm{p}$ value less than 0.05 was accepted as significant.

\section{Results}

Demographic features of fasting and non-fasting groups are demonstrated in Table 1 . No statistically significant difference was detected, when the study groups were compared in terms of age, gender, or body mass index (BMI) $(\mathrm{p}>0.05)$. 
Table I. The demographic characteristics of fasting and non-fasting groups

\begin{tabular}{|l|l|l|c|}
\hline Parameter & Fasting $(\mathbf{n}=\mathbf{3 0})$ & Non-fasting $(\mathbf{n}=\mathbf{2 7})$ & P value \\
\hline Women/man & $13 / 17$ & $16 / 11$ & Ns \\
\hline Age $(\mathrm{mean} \pm \mathrm{SD})$ & $39.23 \pm 16.10$ & $37.2 \pm 14.30$ & Ns \\
\hline BMI $(\mathrm{kg} / \mathrm{m} 2)(\operatorname{mean} \pm \mathrm{SD})$ & $21.12 \pm 3.24$ & $22.18 \pm 2.26$ & Ns \\
\hline
\end{tabular}

As shown in Table II, the TAC level of fasting group was quite high, when compared to the non-fasting group $(\mathrm{p}<0.001)$. On the other hand, TOS level and OSI of non-fasting group were quite high, when compared to the fasting group. ( $\mathrm{p}<=0.019$ and $\mathrm{p}<0.001$ respectively). The oxidative stress index of both groups was shown in Figure 1.

Table II. Comparison of TAC, TOS and OSI levels in fasting and non-fasting groups

\begin{tabular}{|l|l|l|l|}
\hline Parameter & Fasting $(\mathbf{n}=\mathbf{3 0})$ & Non-fasting $(\mathbf{n}=\mathbf{2 7})$ & P value \\
\hline TAC & $1,29 \pm 0,19$ & $1,09 \pm 0,16$ & $<0,001$ \\
\hline TOS & $12,77 \pm 2,23$ & $14,15 \pm 2,04$ & $=0,019$ \\
\hline OSI & $1,01 \pm 0,25$ & $1,33 \pm 0,30$ & $<0,001$ \\
\hline
\end{tabular}

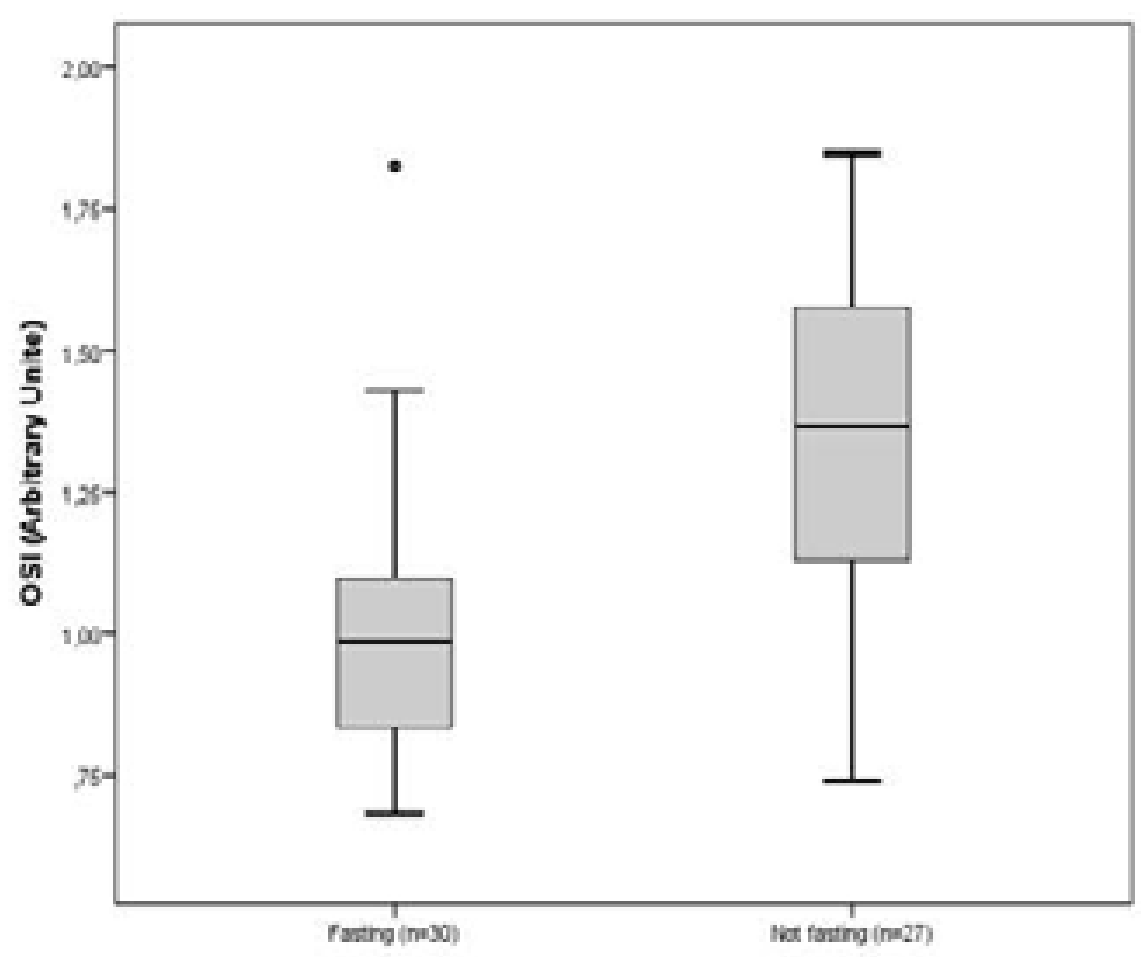

Figure 1. OSI levels in fasting and non-fasting groups 


\section{Discussion}

If oxidative/anti-oxidative balance shifts to the oxidative side, oxidative stress emerges in tissues and organs ${ }^{8}$. Oxidative stress causes production of reactive oxygen species (ROS) and reactive nitrogen species (RNS) such as superoxide radicals, hydrogen peroxide, hydroxyl radicals, singlet oxygen, lipid hydro peroxides, peroxynitrite and other related species?. ROS/RNS can lead to oxidative harm to basic cellular constituents such as membrane lipids, proteins and DNA, which may finally result in cell death $^{10}$. Oxidative harm induced by ROS/RNS is a significant contributing factor in the pathogenesis of aging, cancer, cardiovascular diseases and other degenerative diseases ${ }^{11}$.

Similarly, in our study, the increase of oxidative stress in non-fasting group (Table II and Figure 1) leads to ROS/ RNS increase. As a result, some constituents such as protein, lipid and nucleic acid get harmed, which result in cell death. Consequently, many illnesses might develop. However, by decreasing oxidative stress and increasing TAC, fasting may take a protective role in avoiding many diseases.

In many studies, caloric restriction (CR) is found to protect the onset of some diseases such as: autoimmune diseases, atherosclerosis, cardiomyopathies, cancer, diabetes, renal diseases, neurodegenerative diseases, and respiratory diseases. Therefore, CR has been suggested to have a curative and anti-ageing function ${ }^{12,13}$. Moreover, in many studies, it has also been discussed that CR could delay or protect the onset of many diseases such as cardiovascular diseases, renal diseases, cancers, and diabetes ${ }^{14-17}$.

In our study, it has also been observed that those who fast during Ramadan and therefore have a strict caloric restriction during the day may benefit from similar outcomes. In the fasting group, TAC increase and TOS decrease result in decreasing oxidative stress in tissues and organs. Therefore, any DNA harm, and apoptosis risk could be avoided (Table II, Figure1).

It seems that fasting due to religious motives could regulate oxidative/anti-oxidative balance, delay or prevent the onset of many diseases. Therefore, it might play a significant role in extending life-span.

\section{Conclusion}

Like many other similar studies, our study has found that fasting could regulate oxidative/anti-oxidative balance, It might lead to healthy life.

\section{Conflict of interest}

None declared.

\section{References}

1. The Holy Quran, Surah Al-Baqara, 183rd Verse.

2. (Heysemî, 203 III, 179); (Süyûtî, el-Câmiu's-Sağîr, 2/312, (5060); (Münavî, Feyzul Kadir, 4/21).

3. Iraki L, Bogdan A, Hakkou F, Amrani N, Abkari A, Touitou Y. Ramadan diet restrictions modify the circadian time structure in humans. A study on plasma gastro, insulin, glucose, and calcium and on gastric $\mathrm{pH}$. J Clin Endocrinol Metab. 1997; 82:1261-73.

4. Rouhani MH, Azadbakht L. Is Ramadan fasting related to health outcomes? A review on the related evidence. $J$ Res Med Sci. 2014 Oct; 19(10):987-92. Review

5. Kul S, Savas E, Öztürk ZA, Karadag G. Does Ramadan fasting alter body weight and blood lipids and fasting blood glucose in a healthy population? A meta-analysis. $J$ Relig Health. 2014; 53:929-42.

6. Erel O (2004). A novel automated method to measure total antioxidant response against potent free radical reactions. Clin. Biochem., 2004; 37:112-119.

7. Erel O (2005). A new automated colorimetric method for measuring total oxidant status. Clin. Biochem., 2005; 38: 1103-1111.

8. Halliwell B, Gutteridge JM. Free radicals in biology and medicine, 3rd ed., London: Oxford Science Publications; 1999.

9. Saltman B: Oxidative stress: a radical review. Semin Hematol. 1989; 26: 249-256. PubMed

10. Squadrito GL, Pryor WA: Oxidative chemistry of nitric oxide: the roles of superoxide, peroxynitrite, and carbon dioxide. Free Radic Biol Med. 1998; 25: 392-403. PubMed

11. Sies H: Oxidative stress: oxidants and antioxidants. Exp Physiol. 1997; 82: 291-295. PubMed

12. Imai S: SIRT1 and caloric restriction: an insight into possible trade-offs between robustness and frailty. Curr Opin Clin Nutr Metab Care. 2009, 12:350-6. 
13. Vaquero A, Reinberg D: Calorie restriction and the exercise of chromatin. Genes Dev. 2009, 23:1849-1869. PubMed

14. Mattson MP, Wan R: Beneficial effects of intermittent fasting and caloric restriction on the cardiovascular and cerebrovascular systems. J Nutr Biochem. 2005, 16:129-137.
15. Fontana L, Klein S: Aging, adiposity, and calorie restriction. JAMA. 2007, 297:986-994.

16. Masoro EJ: Overview of caloric restriction and ageing. Mech Ageing Dev. 2005, 126:913-922.

17. Varady KA, Hellerstein MK: Alternate-day fasting and chronic disease prevention: a review of human and animal trials. Am J Clin Nutr. 2007, 86:7-13. 